BULL. AUSTRAL. MATH. SOC.

VOL. $14(1976), 397-398$.

\title{
An addition to a note of mine
}

\section{Kurt Mahler}

A recent improvement by $H$. Guggenheimer of the lower bound for the product of the volumes of a pair of polar reciprocal convex bodies is used to replace one of the results of Kurt Mahler, "Polar analogues of two theorems by Minkowski", Bull. Austral. Math. Soc. 11 (1974), 12l-129 by a best possible one. A similar improvement can be made for the other theorems in my note.

In my note [2], I established three theorems on the connection between symmetric convex bodies in $R^{n}$ and hyperplane lattices in $R^{n}$. The proofs of these theorems were based on the inequality

$$
V(K) V\left(K^{*}\right) \geq n^{-n / 2} \pi^{n} \Gamma((n / 2)+1)^{-2}
$$

for the volumes of a pair of polar reciprocal convex bodies $K$ and $K^{*}$. Recently, Guggenheimer [1] has replaced this inequality by

$$
V(K) V\left(K^{*}\right) \geq 4^{n} / n ! \text {, }
$$

which is best possible; for equality holds if one of the two bodies $K, K^{*}$ is an $n$-dimensional parallelepiped.

On using this improved lower bound in the proofs of my note, but without further changes, my first theorem takes now the following simple form.

THEOREM 1. Let $K: F(x) \leq 1$ be a symmetric convex body of volume

$$
V(K) \geq 2^{n} / n !
$$

Then there exists an integral vector $u \neq 0$ such that $F(x) \geq 1$ at all

Received 11 February 1976. 
real points $x$ satisfying $u \cdot x=1$.

This theorem is best possible as is immediately seen when $K$ is the octahedron

$$
\left|x_{1}\right|+\ldots+\left|x_{n}\right| \leq 1,
$$

and $u$ is the vector $(1,0, \ldots, 0)$.

Theorem 1 implies the following analogue to Minkowski's Theorem on linear forms.

Let

$$
y_{h}=a_{h 1} x_{1}+\ldots+a_{h n} x_{n} \quad(h=1,2, \ldots, n)
$$

be $n$ linear forms with real coefficients of determinant 1 . Then there exist integers $u_{1}, \ldots, u_{n}$ not all zero such that $\left|y_{1}\right|+\ldots+\left|y_{n}\right| \geq 1$ for all real $x_{1}, \ldots, x_{n}$ satisfying $u_{1} x_{1}+\ldots+u_{n} x_{n}=1$.

The same change allows one to improve the other two theorems of my note.

\section{References}

[1] H. Guggenheimer, "Polar reciprocal convex bodies", Israel J. Math. 14 (1973), 309-316.

[2] Kurt Mahler, "Polar analogues of two theorems by Minkowski", Bull. Austral. Math. Soc. 11 (1974), 121-129.

Department of Mathematics, Institute of Advanced Studies, Australian National University, Canberra, ACT. 